\title{
Publication rates: is more better?
}

\author{
R. Gunzburg ${ }^{1}$
}

Received: 11 March 2016/Revised: 11 March 2016/Accepted: 11 March 2016/Published online: 17 March 2016

(c) Springer-Verlag Berlin Heidelberg 2016

In 2005, I looked at some numbers on PubMed for a presentation I had to give at that time. While typing the word 'spine' it generated 86,320 hits. By adding the term 'surgery', this number became 29,983 . Today I did the same and got 181,850 and 79,589 hits, respectively - more than a doubling of the amount of publications over an 11-year span. What does this tell us? That our knowledge increases, or merely that we publish more?

Wei et al. present a study on "worldwide research productivity in the field of spine surgery: a 10-year bibliometric analysis' in this latest issue of the European Spine Journal. Their title, in fact, is a misnomer, for they looked at the research productivity in the field of spine and not only spine surgery. This reflects the profile of most of the publications they analysed. Indeed, Spine, the European Spine Journal and The Spine Journal are multidisciplinary journals dealing with the spine in general, not only surgery. Interestingly, the six most cited articles from the last 10 years are non-surgical papers.

This dramatic increase in number of publications reflects the popularity of spine as a subspecialty in general, and the increase in both surgery rates and available surgical 'techniques' in particular. The wind is in our sails, we are fashionable.

I have my reservations, however, at the quality of what is being produced and published. The number of papers submitted to the European Spine Journal has gone up from
551 in 2005 to 1.919 in 2015. Unfortunately, the quality of the submissions has not gone up correspondingly. Often I see papers that are unnecessary repetitions of previously published studies as researchers may overlook studies, which appeared some decades ago. I am not convinced that this increase in publication rate necessarily leads to a better medicine, improved outcomes and happier patients, or happier health care payers for that matter.

Still too many papers are retrospective and of poor design, or with too many confounding factors. Whereas the gold standard of 'controlled randomized trial' remains one to strive for, in spite of possible ethical concerns, valuable alternatives such as papers emanating from registries gain field.

Wei et al. found that China and Korea show the most dramatic increase in publication rate. To guide researchers in that part of the world plan their studies well and thus increase their chance of acceptance for publication, the European Spine Journal is holding a workshop on 'How to increase the chance for acceptance of a publication' during the upcoming Spine Week conference which is being held in Singapore from May 16-20, 2016 (http://www.spine week2016.org).

\section{Compliance with ethical standards}

Conflict of interest None.
R. Gunzburg

robert@gunzburg.be

1 Cavell Spine Centre, Edith Cavell Clinic, Edith Cavell Street 32, 1180 Brussels, Belgium 